\title{
Identification of the replication region in pBCNF5603, a bacteriocin-encoding plasmid, in the enterotoxigenic Clostridium perfringens strain F5603
}

\author{
Kazuaki Miyamoto ${ }^{\text {* }}$, Soshi Seike ${ }^{1}$, Teruhisa Takagishi ${ }^{1}$, Kensuke Okui ${ }^{1}$, Masataka Oda², Masaya Takehara ${ }^{1}$ \\ and Masahiro Nagahama' ${ }^{1}$
}

\begin{abstract}
Background: Most recent studies of Clostridium perfringens plasmids have focused on toxin-encoding or antibiotic resistance plasmids. To cause intestinal disease, a toxigenic strain must grow in the intestines to levels allowing for sufficient toxin production and this in vivo growth often involves overcoming the normal intestinal microbial population. For this purpose, bacteriocin production might be important.

Results: In this study, as the first step in the genetic analysis of a co-existing plasmid with an enterotoxin gene (cpe)-encoding plasmid, the bacteriocin gene-encoding plasmid, pBCNF5603, was completely sequenced. This plasmid has some homology with two previously sequenced C. perfringens plasmids, namely, pCP13 carrying a cpb2 gene and plP404 carrying a bcn gene. Using recombinant plasmids, the rep gene homologous to the PCP63 gene on pCP13 appeared to be functional. Comparative genomics indicated that the identified rep gene homologs were found on two additional toxin plasmids, PCP-OS1 and PCP-TS1. While functional analysis using recombinant plasmids indicated that pBCNF5603 and pCP13 are likely to be incompatible, the plasmid replication and partitioning region of pBCNF5603 alone was insufficient for stable maintenance of this plasmid.

Conclusions: These findings suggest that pBCNF5603 evolved from recombination events between C. perfringens plasmids and inter-species mobile genetic element(s). In addition, the bcn-encoding plasmid, pBCNF5603, is likely to be included in the Inc family, which includes PCP13 and two variant iota-encoding plasmids. Furthermore, the bcn gene on pBCNF5603 could contribute to gastrointestinal disease induced by enterotoxigenic C. perfringens.
\end{abstract}

Keywords: Clostridium perfringens, Bacteriocin gene, rep gene, Plasmid compatibility, Plasmid stability

\section{Background}

Clostridium perfringens is an important Gram-positive pathogen, but also a member of the normal gastrointestinal flora of humans and animals [1]. The pathogenicity of this bacterium to humans and livestock largely depends upon toxin production, with 16 different C. perfringens toxins reported in the literature [1]. Most $C$. perfringens toxin genes, including three typing toxin $(\beta, \varepsilon, \iota)$ genes and recently identified novel toxin genes,

\footnotetext{
*Correspondence: kazupitts@jtw.zaq.ne.jp

'Department of Microbiology, Faculty of Pharmaceutical Sciences, Tokushima Bunri University, Yamashiro-cho 180, Tokushima 770-8514, Japan Full list of author information is available at the end of the article
}

such as necrotic enteritis B-like toxin gene (netB) and toxin C. perfringens large cytotoxin gene (tepL), are present on plasmids [2-4]. Therefore, the presence of toxin geneencoding plasmids is crucial for $C$. perfringens virulence.

For $C$. perfringens strains producing C. perfringens enterotoxin (CPE) to cause non-foodborne human intestinal disease, they not only have to produce toxins, but also must overcome the normal microbial population so they can grow to pathogenic levels [5]. For this purpose, the production of bacteriocins might be useful [5]. Previous surveys of bacteriocins support this possibility since studies conducted in the 1960s and 1970s showed that many C. perfringens isolates produce bacteriocins [6]. 
However, a bacteriocin of $C$. perfringens has received much less attention recently than the toxins which induce the diseases caused by this bacterium, such as human and veterinary diarrhea.

On a completely sequenced cpe-encoding plasmid, pCPF5603, in the enterotoxigenic F5603 strain, which was isolated from a patient suffering from non-foodborne diarrheal disease, the gene encoding a bacteriocin that contributes to $C$. perfringens virulence was not identified. Therefore, an unidentified virulence factor such as a bacteriocin could be encoded on another co-existing plasmid.

In this study, the plasmid carrying a bacteriocin gene (bcn) homolog in the enterotoxigenic F5603 strain was completely sequenced. Thereafter, to understand the relationships with the co-existing prototype of toxin-encoding plasmid or other type(s) of toxin plasmid such as pCP13, the replication region of $b c n$-encoding plasmid was identified. Then, the replication region on pBCNF5603 was compared with those of other sequenced plasmids by comparative genomics because the replication region, which must contain the replication initiation protein (Rep) gene (rep), is a basic element of a plasmid [7]. Moreover, the identified replication and putative partitioning region was functionally investigated.

\section{Methods}

\section{Bacterial strains and media}

Clostridium perfringens strains F5603, NCTC8533, ATCC3624, and strain 13 were used in this study [8-10]. For the growth of these strains, TGY medium (3\% tryptic soy broth [TSB] [BD Bioscience, Tokyo, Japan], $2 \%$ glucose, $1 \%$ yeast extract [BD Bioscience, Tokyo, Japan], and 0.1 \% L-cysteine [Wako Pure Chemical, Osaka, Japan]) with or without chloramphenicol $(10 \mu \mathrm{g} / \mathrm{ml}$ ) (Wako Pure Chemical, Osaka, Japan) was used as previously described $[8,9]$. E. coli HB101 was used as the host for the recombinant plasmids used for DNA sequencing or for constructing recombinant plasmids carrying the putative replication region from $C$. perfringens plasmids. For the selection of $E$. coli or $C$. perfringens transformants, TSB agar containing ampicillin (100 $\mathrm{\mu g} / \mathrm{ml}$ ) (Wako Pure Chemical, Osaka, Japan) or chloramphenicol $(30 \mu \mathrm{g} / \mathrm{ml})$ and Brain Heart Infusion (BHI) (BD Bioscience, Tokyo, Japan) agar with chloramphenicol $(15 \mu \mathrm{g} / \mathrm{ml})$ were used, respectively.

\section{Complete sequencing of the bacteriocin-encoding plasmid pBCNF5603 in F5603 and plasmid pCP8533512 carrying a pIP404-rep homolog in NCTC8533}

Sequencing of a bacteriocin-encoding plasmid in strain F5603 was performed using a previously described method [8]. Briefly, crude plasmid DNA preparations were prepared as described previously [8] and then transformants carrying F5603 plasmid DNA fragments, which were not from the completely sequenced cpe-encoding plasmid pCPF5603, were selected and then sequenced. To complete the sequencing of pBCNF5603, long-range PCR analysis was performed using primers based upon endsequence information. Those PCR products were then sequenced using a primer walking strategy.

For sequencing of a small plasmid named pCP8533S12, which is present in type B strain NCTC8533 and carries a pIP404 rep homolog, PCR primers were constructed based on the sequence of a small plasmid in ATCC3626, another type B strain (accession number: NZ_ABDV01000054). The PCR products obtained using those primers were then used for direct sequencing.

Putative ORFs on both sequenced plasmids were predicted with ORF finder software (http://www.ncbi.nml.nih. gov/gorf/gorf.html) and a putative Shine-Dalgarno sequence for each ORF was identified. Annotation of each ORF was predicted with BLAST blast.ncbi.nlm.nih.gov/ Blast.egi web database software.

\section{Identification of the replication region in the bacteriocin- encoding plasmid pBCNF5603}

Sequence analysis with BLAST indicated that pBCNF5603 mainly consists of two regions: a pCP13-like ORF region and a pIP404-like bacteriocin region (described in the results section). In addition, each region of pBCNF5603 was shown to harbor putative replication regions, namely, the PBCN16-18 region homologous to the PCP63-parA (soj)parB (spoOJ) gene cluster on pCP13 and the PBCN29-30 region homologous to the cop-rep region of pIP404. To investigate the replication ability of these two putative replication regions, each one was separately amplified by PCR. The primers used for amplification of the putative replication regions are listed in Additional file 1: Table S1. In these PCR reactions, each PCR mixture contained $1 \mu \mathrm{l}$ of template DNA, $0.4 \mu \mathrm{l}$ of GXL Taq polymerase (TAKARA BIO, Otsu, Japan), $3 \mu \mathrm{l}$ of $2 \mathrm{mM}$ NTPs, $10 \mu \mathrm{l}$ of PCR buffer, and $2 \mu \mathrm{l}$ of each primer pair $(1 \mu \mathrm{M}$ final concentration). PCR reactions were performed under conditions previously described [11]. After confirming PCR amplification, each PCR product was clarified using the QIAquick PCR purification kit (QIAGEN, Tokyo, Japan) and then digested with restriction enzymes: AvrII/BstBI (NEB, Tokyo, Japan) or AvrII/HpaI (NEB, Tokyo, Japan) for the $\mathrm{pCP} 13$-homologous putative replication region on pBCNF5603; SpeI/SnaBI (NEB, Tokyo, Japan) for the pIP404-homologous putative replication region on pBCNF5603; and BsmI/PflMI (NEB, Tokyo, Japan) for the putative rep region on pCP8533S12 (Fig. 2). Digested DNA fragments were subjected to filling with Klenow fragment polymerase (Promega, Tokyo, Japan), and were ligated into a fragment of shuttle vector pJIR750, in which the rep gene of pIP404 had been removed by digestion with XmnI (NEB, Tokyo, Japan) and SpeI (NEB, Tokyo, Japan) [12]. 
The resultant plasmids, named pKZ100, pKZ110, pKZ200, or pKZ210, were transformed into E. coli HB101 and then electroporated into transformable $C$. perfringens type A strains, ATCC3624 and strain 13, using Electro Cell Manipulator, ECM630 (BTX), according to the manufacturer's instructions (Table 1) [13]. Briefly, $0.6 \mathrm{ml}$ of overnight TGY culture of C. perfringens strain was inoculated into $20 \mathrm{ml}$ of TGY medium and anaerobically incubated at $37^{\circ} \mathrm{C}$ for 6 to $8 \mathrm{~h}$. Bacterial cells were collected by centrifugation at 3,000 x g for 20 min. Collected cells were washed twice with SMP solution (270 $\mathrm{mM}$ sucrose [Pure Chemical, Osaka, Japan], 1 $\mathrm{mM} \mathrm{MgCl} 2$ [Pure Chemical, Osaka, Japan] in $7 \mathrm{mM}$ sodium phosphate solution, pH7.4) and then centrifuged. The washed bacterial pellet was re-suspended with 1.2 ml of SMP solution. A $600 \mu \mathrm{l}$ aliquot of re-suspended cells was used for electroporation. Transformants carrying a recombinant plasmid were selected on BHI agar plates containing $15 \mu \mathrm{g} / \mathrm{ml}$ chloramphenicol.

\section{Compatibility assay between recombinant plasmids derived from pBCNF5603 and pCP13}

From the results of comparative genomics, the identified rep gene was highly similar to PCP63 (probable rep gene on $\mathrm{pCP} 13$ ) in strain 13. To understand the relationship between pBCNF5603 and pCP13, compatibility assay was performed. The constructed recombinant plasmid (pKZ200 or pKZ210) carrying the rep gene of pBCNF5603 was introduced into strain 13 according to the electroporation methods previously described [13]. In the final step of the electroporation procedures, electroporated samples were inoculated into $4 \mathrm{ml}$ of pre-warmed TGY medium without antibiotics. After 3-h incubation at $37{ }^{\circ} \mathrm{C}, 0.2 \mathrm{ml}$ of a $4 \mathrm{ml}$ transformed culture sample was inoculated into $10 \mathrm{ml}$ of TGY medium with chloramphenicol $(10 \mathrm{mg} / \mathrm{ml})$ and incubated at $37{ }^{\circ} \mathrm{C}$. The next day, $0.2 \mathrm{ml}$ of culture sample was inoculated in fresh TGY medium with chloramphenicol and incubated at $37{ }^{\circ} \mathrm{C}$ (passage 2). One-day culture of this second-passage sample was spread onto a BHI agar plate containing $15 \mathrm{mg} / \mathrm{ml}$ chloramphenicol, which was then anaerobically incubated at $37^{\circ} \mathrm{C}$.

To investigate the presence or absence of pCP13 in transformed C. perfringens colonies, randomly selected chloramphenicol-resistant colonies were tested by colony PCR assay for three genes: $c p b 2$ (the $\beta 2$ toxin gene),
topA (the gene of topoisomerase homolog), and cna (a putative collagen adhesion protein gene), on pCP13. For colony PCR reaction, colonies were suspended in $20 \mu \mathrm{l}$ of distilled water and then heated at $100{ }^{\circ} \mathrm{C}$ for $10 \mathrm{~min}$. After brief centrifugation, $1 \mu$ l of supernatant was used as a DNA template with PCR reaction. Each PCR mixture $(50 \mu \mathrm{l})$ contained $1 \mu \mathrm{l}$ of template DNA, $0.25 \mu \mathrm{l}$ of EX Taq polymerase (TAKARA BIO, Otsu, Japan), $4 \mu \mathrm{l}$ of $2.5 \mathrm{mM}$ NTPs, $5 \mu \mathrm{l}$ of PCR buffer, and $2 \mu \mathrm{l}$ of each primer pair ( $1 \mu \mathrm{M}$ final concentration). PCR reactions were performed under the following conditions: $95{ }^{\circ} \mathrm{C}$, for 2 min; 35 cycles of $95{ }^{\circ} \mathrm{C}$ for $30 \mathrm{sec}, 61^{\circ} \mathrm{C}$ (for cpb2, cna) or $64{ }^{\circ} \mathrm{C}$ (for topA) for $30 \mathrm{sec}$, and $72{ }^{\circ} \mathrm{C}$ for $30 \mathrm{sec}$; and a single extension at $72{ }^{\circ} \mathrm{C}$ for $5 \mathrm{~min}$. PCR products were electrophoresed on a $1.5 \%$ agarose gel followed by staining with UltraPower ${ }^{\mathrm{TM}}$ DNA Stain (Gellex, Tokyo, Japan), and then fluorescence was detected using LAS-4000 (FUJIFILM).

\section{The stability of the constructed recombinant plasmid in two type A C. perfringens strains}

Upstream of the rep gene, pBCNF5603 is also assumed to harbor the conserved plasmid partitioning region, including parA and parB genes and putative parS sequences. To investigate the contribution of putative plasmid partitioning regions to plasmid stability, the stability of a recombinant plasmid, pKZ200, which carries the replication and putative partitioning region, was estimated (Fig. 2). First, the recombinant plasmid was introduced into two transformable $C$. perfringens strains: ATCC3624 and strain 13. The type A ATCC3624 strain is likely to carry no plasmid, but strain 13 harbors pCP13, which carries a replication and partitioning region homologous to that of pBCNF5603, as described in the results section. Therefore, to estimate the stability of pKZ200 in strain 13, two chloramphenicol-resistant pCP13-free clones of strain 13-derived transformants were selected using the compatibility assay described above, and then used for plasmid stability assay; specifically, two clones of ATCC3624-derived transformants and two clones of pCP13-free strain 13 transformants were used.

Each of the stocked clones in cooked meat medium was inoculated into $10 \mathrm{ml}$ of FTG medium (BD Bioscience, Tokyo, Japan) and incubated at $37{ }^{\circ} \mathrm{C}$. Overnight cultures were then inoculated into $10 \mathrm{ml}$ of TGY medium, incubated

Table 1 Plasmids constructed and used in this study

\begin{tabular}{ll}
\hline Plasmid & \\
\hline pJIR750 & Hindll//ECoRV (2629 bp) fragment of plP404 replication region \\
pKZ100 & pJIR750 (Xmnl/Spel) $\Omega$ pBCNF5603 PCR product (Spel/SnaBl; 3120 bp) (cop and rep fragment) \\
pKZ110 & pJIR750 (Xmnl/Spel) $\Omega$ pCP8533S12 PCR product (Bsml/PflMl; 2632 bp) (rep fragment) \\
pKZ200 & pJIR750 (Xmnl/Spel) $\Omega$ pBCNF5603 PCR product (Avrll/BstBl; 5476 bp) (PCP63, soj, parB and PCP03 fragment) \\
pKZ210 & pJIR750 (Xmnl/Spel) $\Omega$ pBCNF5603 PCR product (Avrll/Hpal; 2271 bp) (PCP63 fragment)
\end{tabular}


at $37^{\circ} \mathrm{C}$, then spread onto a BHI agar plate with chloramphenicol $(15 \mathrm{mg} / \mathrm{ml})$, and then anaerobically incubated at $37^{\circ} \mathrm{C}$. To estimate the stability of the recombinant plasmid, pKZ200, in C. perfringens strains, two separate colonies of each clone were inoculated into $10 \mathrm{ml}$ of TGY medium cotaining chloramphenicol $(10 \mu \mathrm{g} / \mathrm{ml})$ and incubated at $37{ }^{\circ} \mathrm{C}$. Then, $0.2 \mathrm{ml}$ of overnight culture was inoculated into $10 \mathrm{ml}$ of fresh TGY medium containing chloramphenicol. After incubation for $24 \mathrm{~h}$ at $37^{\circ} \mathrm{C}$, a 10 $\mu \mathrm{l}$ culture sample was transferred to $10 \mathrm{ml}$ of fresh TGY without chloramphenicol, so that approximately 10 generations were obtained per growth cycle of $24 \mathrm{~h}\left(1: 2^{10}\right.$ dilution rate) [14]. These inoculated cultures were used as a first passage. Every $24 \mathrm{~h}, 10 \mu \mathrm{l}$ of the full-grown cultures was transferred to $10 \mathrm{ml}$ of fresh TGY. After 6 passages, the cultures were diluted and plated on a BHI plate without chloramphenicol. Determination of the fraction of recombinant plasmid cured cells in the population was performed by replica-picking of 25 randomly chosen colonies onto BHI with chloramphenicol $(15 \mu \mathrm{g} / \mathrm{ml})$ and without chloramphenicol, and scoring the chloramphenicol-sensitive colonies. To confirm the presence or absence of pKZ200, eight chloramphenicolsensitive and eight chloramphenicol-resistant colonies were tested by colony PCR assay for the rep gene. Incompatibility between pKZ200 and indigenous pCP13 must influence the stability of the recombinant plasmid. To avoid the possibility of contamination with original strain 13, the same sixteen colonies were tested by $c p b 2$-PCR assay.

\section{Results}

Organization of the bcn5603-encoding plasmid pBCNF5603 in F5603

In a previous study with pulsed-field gel electrophoresis (PFGE) analysis, C. perfringens F5603 strain carried two large plasmids, $\sim 75 \mathrm{~kb}$ cpe-encoding plasmid and another $\sim 40 \mathrm{~kb}$ plasmid [8] (Additional file 2: Figure S1). Sequencing revealed that pBCNF5603 (GenBank accession no. AB189671) is 36,695 bp in size, with $25.4 \%$ GC content and encoding 36 ORFs. The size of sequenced plasmid closely agreed with the predicted size of a smaller $40 \mathrm{~kb}$ plasmid in F5603 strain in PFGE analysis. In comparison with the sequences of $\mathrm{pCP} 13$ and pIP404, pBCNF5603 carries 15 or 8 ORFs that are highly homologous to ORFs present on pCP13 and pIP404, respectively [10, 15] (Fig. 1) (Additional file 1: Table S2). Of the fourteen pCP13 ORF homologs on pBCNF5603, a gene cluster from $\mathrm{PBCN} 8$ to $\mathrm{PBCN} 15$ was similarly arranged from PCP34 to PCP44, and a gene cluster from PBCN16 to PBCN19 was also from PCP63 to PCP03 (Fig. 1) (Additional file 1: Table S2) [10]. In the gene cluster from PBCN16 to PBCN19, pBCNF5603 carries parA and parB genes, which are highly homologous to the respective genes on $\mathrm{pCP} 13$, but which are absent from pIP404 $[10,15]$. Therefore, this region might contain the genes for plasmid replication and maintenance, while the replication system of pCP13 has not yet been identified [10].

In eight shared ORFs with pIP404, pBCNF5603 encodes the putative bacteriocin BCN5603 gene (bcn5603) and upstream UV-inducible regulation genes, uviA and

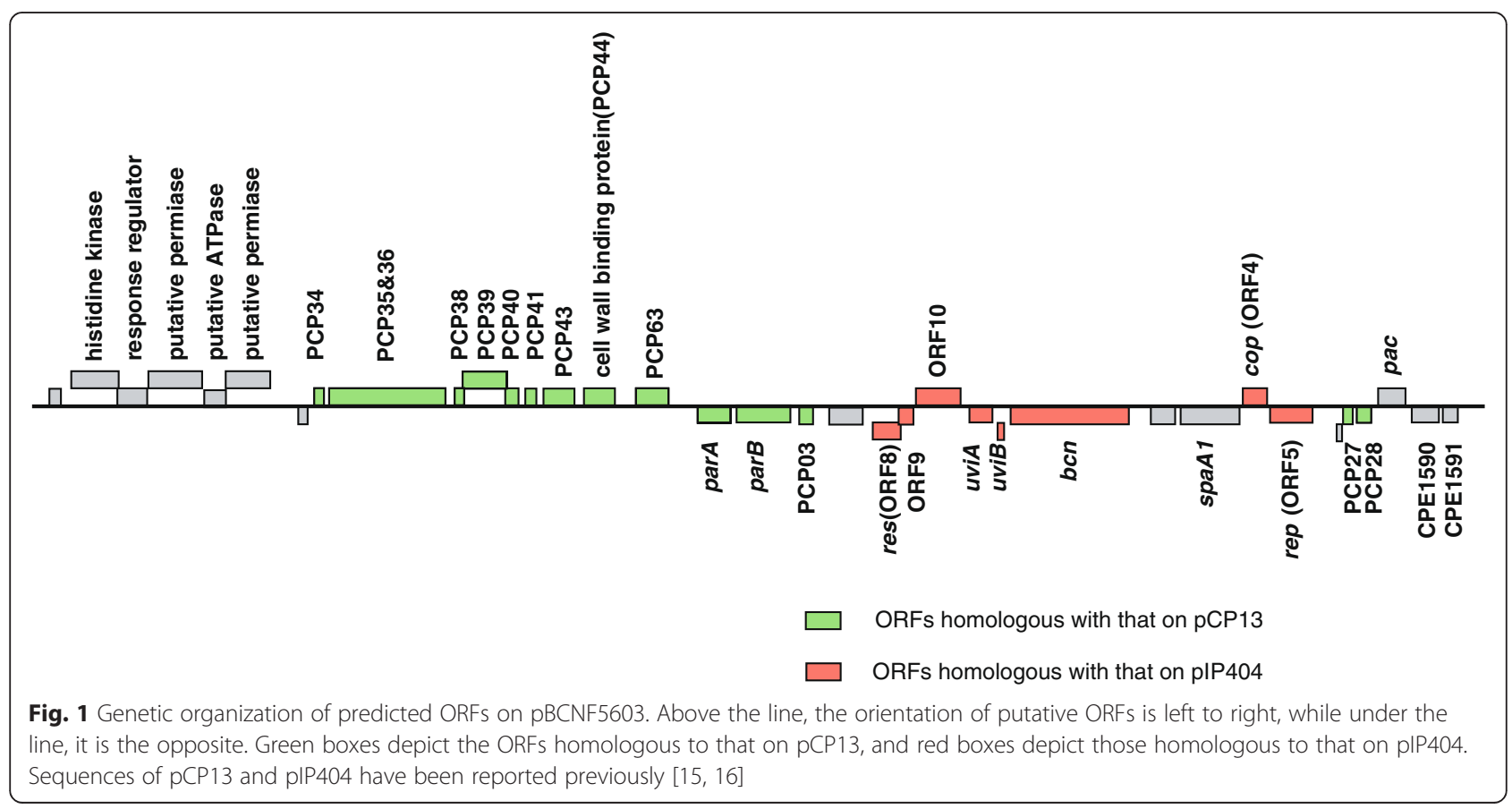


uvi $\mathrm{B}$, which are also present on pIP404 [16]. The BCN5 gene $(b c n)$ was originally found in the type A human isolate CPN50. In comparative genomics, the product of the $b c n 5603$ gene, BCN5603, showed similarity to the gene products in $C$. perfringens strains: food poisoning strain-derived SM101, type E JGS1987, type C JGS1495, and type D JGS1721 strains, in addition to CPN50 strain. These bacteriocins in the virulent $C$. perfringens strain shared conserved domains: two SH3b domains (usually found on bacteriocins) and peptidase M14 superfamily domain, which contained two Zn-binding sites. In addition to these conserved domains on putative bacteriocins, BCN5603 carried putative cell wall binding repeat, and two glucan-binding repeats (Glucan65).

Of the bacteriocins in $C$. perfringens, the regulation of BCN5 production has been investigated most intensively; BCN5 is induced by an SOS response to UV light or mitomycin $C$ treatment $[16,17]$. Of the putative bacteriocin regulatory genes, the putative $u v i A$ gene on pBCNF5603 has $98.9 \%$ nucleotide sequence homology with the uviA gene, which is the putative sigma factor for the $b c n 5$ gene on pIP404. In addition, on the basis of amino acid substitution of the uviA genes on both plasmids, regions 4.2 and 2.4 of the UviA sigma factor, which are implicated in interactions with the -35 and -10 sequence of the promoter, were completely identical [16]. The $u v i B$ gene on pBCNF5603 had a completely identical nucleotide sequence with the $u v i B$ gene, which is the putative bacteriocin immunity-related gene on pIP404 [16]. Moreover, the bcn5603-uviA-uviB gene cluster had three promoter sequences almost identical to those in the original $b c n$ gene region on pIP404. Specifically, compared with the matching promoter sequences of BCN5 [17], the bcn5603 gene has two putative promoter sequences: P1 (the strongest promoter of the $b c n$ gene on pIP404) and P2, which are completely identical, as well as P3 with only one nucleotide change. Therefore, the bcn5603 gene might be dependent on UviA for activation. Furthermore, the P4 and P5 promoters upstream of the uviA gene on pBCNF5603 had identical sequences to those on pIP404, and the putative repressor LexA-binding site of the $b c n 5603$ gene also had an identical sequence with the corresponding site in the pIP404 BCN5 regulatory region [17]. From these highly similar sequences in the regulatory region, the enterotoxigenic strain F5603 might produce BCN5603 in a similar manner to BCN5 by strain CNP50.

pBCNF5603 also carries a cluster of five ORFs (putatively encoding a sensor histidine kinase, response regulator, ATPase, and two permease genes) with high homology to respective genes present on the chromosome of E. faecalis V583 and the S. agalactiae 2603V/R strain [18, 19] (Fig. 1) (Additional file 1: Table S2).

Overall, pBCNF5603 was identified as a bacteriocinencoding plasmid that developed from a mosaic fusion of C. perfringens plasmids, $c p b 2$-encoding $\mathrm{pCP} 13$ and $b c n$-encoding pIP404, and a foreign gene cluster.

\section{Identification of the replication region of pBCNF5603}

Completely sequenced pBCNF5603 is likely to be an important plasmid for $C$. perfringens virulence because this plasmid encodes a bacteriocin gene. Moreover, pBCNF5603 co-exists with one of the prototype cpe-encoding plasmids, pCPF5603. Therefore, to investigate further the bacteriocin gene-encoding plasmid, the replication region of pBCNF5603 was initially identified in the current study. Sequencing and comparative genomics showed that pBCNF5603 carried two sets of potential plasmid replication regions, namely, the region containing putative plasmid partitioning genes, parA and parB, which was similar to that region on pCP13 (Fig. 1), and putative rep and cop genes similar to the replication region of pIP404.

It was initially shown that a recombinant plasmid named pKZ200 carrying a $\sim 5.5 \mathrm{~kb}$ fragment including the PBCN16 (PCP63 homolog)-parA-parB-PBCN19 (PCP03 homolog) gene cluster could replicate in $C$. perfringens strains, ATCC3624 and strain 13 (Fig. 2a, Table 1). The recombinant plasmid that still supported plasmid replication in two strains was pKZ210, carrying a smaller $\sim 2.3 \mathrm{~kb}$ region containing $\mathrm{PBCN} 16$, and its upstream region (including the partial parA ORF) (Fig. 2a, Table 1). These results indicate that PBCN16, the product of which is a homolog of the PCP63 product, was likely to be the rep gene of pBCNF5603. The upstream region of the identified rep gene, five distinct pairs of 6 to 8 bp inverted repeats (IRs), which could act as iteron-like sequences in the initiation of pBCNF5603 replication, and an AT-rich region, which is also important for plasmid replication, were identified (Fig. 3) [7]. Therefore, pBCNF5603 was identified as one of the iteron-containing plasmids (ICP) in C. perfringens.

Concerning the replication ability of the second region, ATCC3624 strain carrying the constructed plasmid named pKZ100, which carried the putative cop-rep region similar to the replication region of pIP404, was not detected in several electroporation experiments (Table 1, Fig. 2b). On the other hand, a constructed plasmid named pKZ110 carrying the $\sim 2.6 \mathrm{~kb}$ region containing the putative rep gene of pCP8533S12 could replicate in the ATCC3624 strain (Table 1, Fig. 2b).

Collectively, only the region containing genes similar to the genes on pCP13 was identified as a region carrying the newly identified rep gene, while two potential replication (and maintenance) regions were identified on pBCNF5603 by comparative genomics.

\section{Comparative genomic analysis of the genes in the identified replication region}

By comparative genomics using the GenBank database, plasmids carrying a homolog of the identified rep gene 


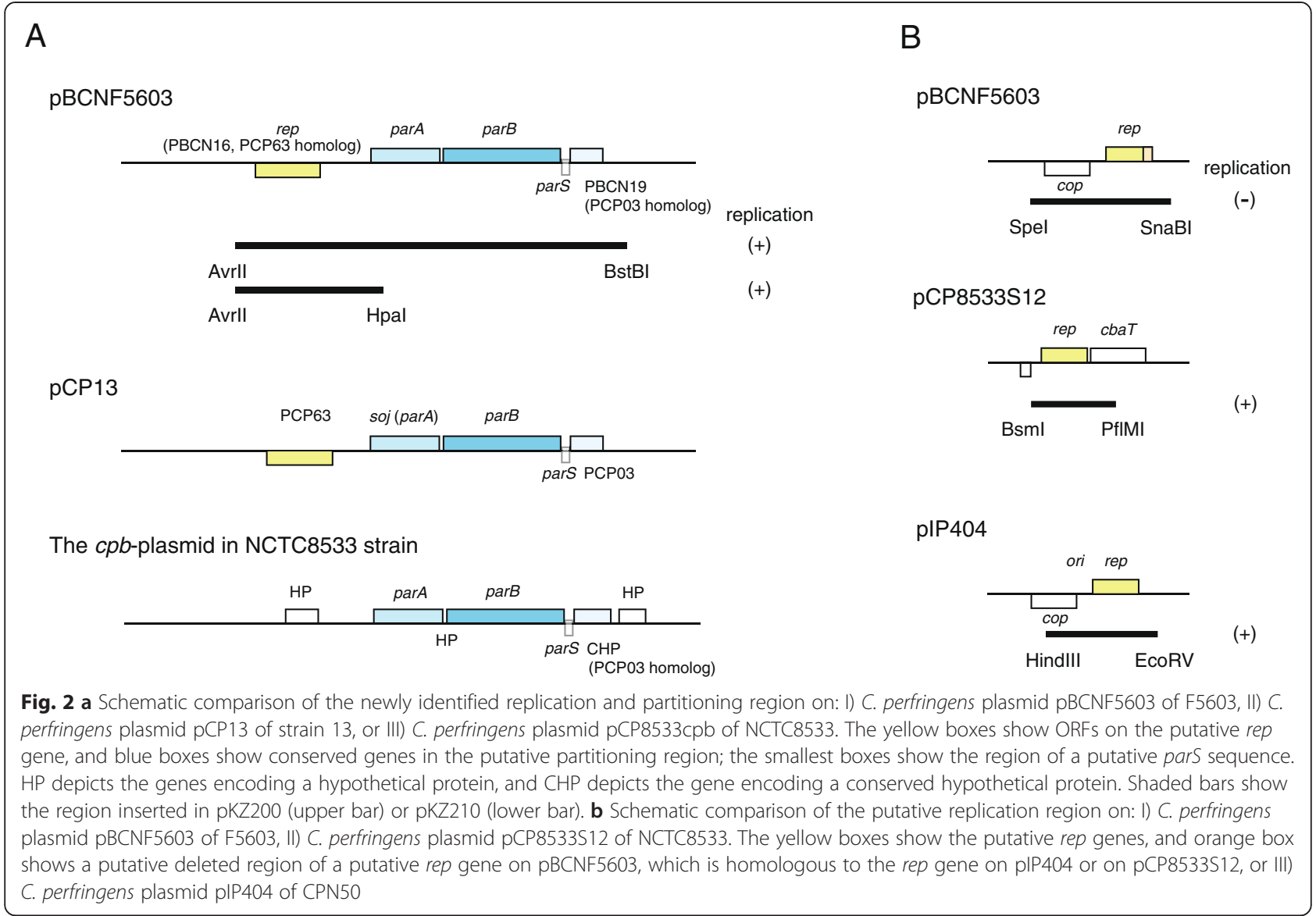

of pBCNF5603 were investigated. In addition to PCP63 on pCP13 (accession number: AP003515), four other homologous genes were found, namely, a gene on pCPOS1 (accession number: AP013033), a gene on pCP-TS1 (accession number: AP013034) (these two plasmids harbor the recently identified iota toxin gene variants), a gene on pF262B (accession number: AFES01000051), and a gene on a plasmid in the WAL-14572 strain (accession number: ADLP01000024) [20, 21] (Table 2). These genes encode hypothetical proteins. Of these genes, PCP63 (651 bp) on pCP13 was the smallest; the rep gene on pBCNF5603 was 852 bp long, and the homologous genes on pCP-TS1 or pCP-OS1 and those of pF262B or plasmid in WAL-14572 were 918 bp and 906 $\mathrm{bp}$, respectively. In the upstream region, each of the putative rep genes on the four plasmids carried iteron-like sequences that were highly similar to those of the rep gene of pBCNF5603, while the putative iteron sequences were not identified in the upstream region of PCP63 (Fig. 3). Moreover, pBCNF5603 and pCP13 in strain 13 from our laboratory stocks were likely to be incompatible (described in the results section). Therefore, for further comparison of those homologs, sequencing analysis of the putative replication region of pCP13 in strain 13 in our laboratory stocks was performed. In terms of the sequencing results of the PCP63 region in strain 13 from our laboratory stocks, PCP63 was 906 bp long and also harbored highly similar iteron-like sequences with a putative rep gene on other plasmids (accession number: LC011526) (Fig. 3, Table 2).

Collectively, bacteriocin- or toxin-encoding plasmids harbor the rep gene homologous to that of pBCNF5603; the plasmid group carrying the pBCNF5603-type rep gene is likely to contribute to $C$. perfringens virulence.

Sequencing also showed that pBCNF5603 carries several gene homologs that appear to be involved in the replication and maintenance systems of pIP404 [15]. The replication system of pIP404 is similar to the Inc18 family of plasmids, whose replication system consists of cop-repori-res genes [15]. Of these four replication component genes, pBCNF5603 was found to carry two ORFs, namely, PBCN29 and PBCN30, which are highly similar to pIP404 genes that are thought to encode putative plasmid stability and replication factors, respectively [15]. Therefore, a candidate Rep protein of pBCNF5603 is probably encoded by PBCN30, which is homologous (92\% identity) to the rep gene (ORF5) of pIP404 (Additional file 1: Table S1). However, PBCN30, which is $\sim 1,000$ bp in size, is smaller than 


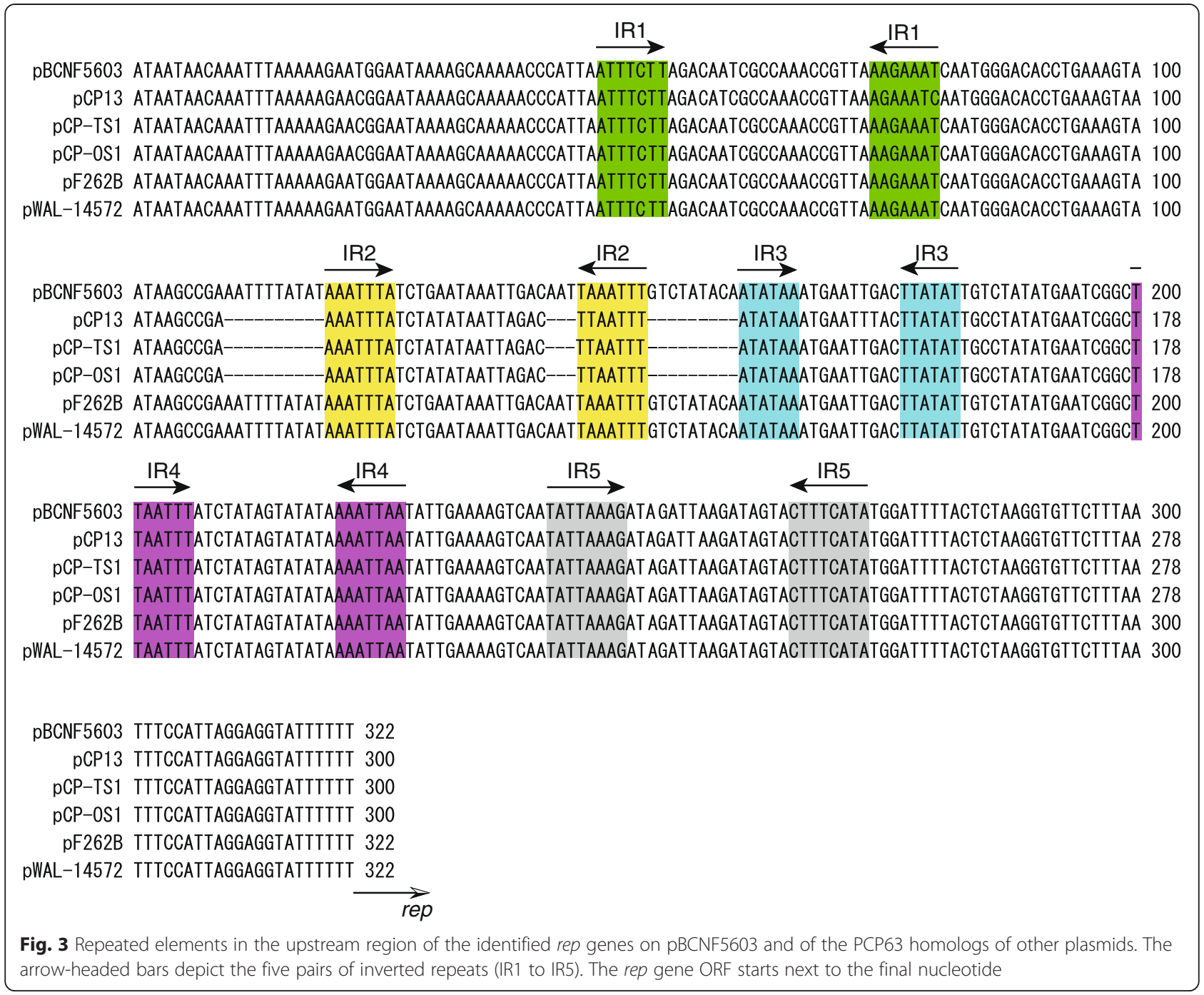

Table 2 Similarity of the genes on the replication and partitioning region among C. perfringens plasmids carrying PCP63 homolog

\begin{tabular}{|c|c|c|c|c|c|c|c|c|c|}
\hline & \multicolumn{2}{|c|}{ PCP63 homologs } & \multicolumn{2}{|l|}{ parA (soj) } & \multicolumn{2}{|l|}{ parB } & \multirow{2}{*}{$\begin{array}{l}\text { pars sequence } \\
\text { length }\end{array}$} & \multicolumn{2}{|c|}{ PCP03 homologs } \\
\hline & ORF size & Identity $^{\mathrm{a}}$ & ORF size & Identity $^{\mathrm{a}}$ & ORF size & Identity $^{a}$ & & ORF size & Identity ${ }^{c}$ \\
\hline pBCNF5603 & 852 bp & $100 \%$ & $753 \mathrm{bp}$ & $100 \%$ & $1278 \mathrm{bp}$ & $100 \%$ & $117 \mathrm{bp}$ & $363 \mathrm{bp}$ & $100 \%$ \\
\hline pCP13 & 906 bp & $92 \%$ & $753 \mathrm{bp}$ & $99 \%$ & $1281 \mathrm{bp}$ & $96 \%$ & $117 \mathrm{bp}$ & $363 \mathrm{bp}$ & $94 \%$ \\
\hline pCP-TS1 & 918 bp & $91 \%$ & $753 \mathrm{bp}$ & $97 \%$ & $1281 \mathrm{bp}$ & $99 \%$ & $117 \mathrm{bp}$ & $363 \mathrm{bp}$ & $90 \%$ \\
\hline pCP-OS1 & 918 bp & $90 \%$ & $753 \mathrm{bp}$ & $97 \%$ & 1281 bp & $99 \%$ & $117 \mathrm{bp}$ & $363 \mathrm{bp}$ & $90 \%$ \\
\hline pF262B & 906 bp & $93 \%$ & $753 \mathrm{bp}$ & $99 \%$ & 1278 bp & $100 \%$ & $117 \mathrm{bp}$ & $363 \mathrm{bp}$ & $99 \%$ \\
\hline pWAL-14572 & $906 \mathrm{bp}$ & $93 \%$ & $753 \mathrm{bp}$ & $99 \%$ & $1278 \mathrm{bp}$ & $100 \%$ & $117 \mathrm{bp}$ & $363 \mathrm{bp}$ & $99 \%$ \\
\hline pCP8533cpb & - & - & $753 \mathrm{bp}$ & $98 \%$ & 1281 bp & $96 \%$ & $117 \mathrm{bp}$ & $363 \mathrm{bp}$ & $96 \%$ \\
\hline JGS1945cpb & - & - & $753 \mathrm{bp}$ & $99 \%$ & 1281 bp & $96 \%$ & $117 \mathrm{bp}$ & $363 \mathrm{bp}$ & $95 \%$ \\
\hline
\end{tabular}

a: Substitutional amino acid identity 
pIP404 ORF5 ( 1,200 bp in length) by approximately 200 bp in the 3 ' region (Fig. 2b).

A putative rep gene, which is also similar to the rep gene on pIP404, was also found on a small plasmid $(\sim 12 \mathrm{~kb})$ in type B strain NCTC8533 and named pCP8533S12 (accession number: AB736082, Additional file 1: Table S3) (Fig. 2b). The size of this rep gene was similar to that of the rep gene on pIP404, but larger than that of the $\mathrm{PBCN} 30$ gene on pBCN5603. In the GenBank database, homologous rep genes were found on a $\sim 12 \mathrm{~kb}$ plasmid in type B strain ATCC3626 (accession number: ABDV01000054), on a $\sim 13 \mathrm{~kb}$ plasmid in type $\mathrm{C}$ strain JGS1945 (accession number: ABDU01000032), on a $\sim 4 \mathrm{~kb}$ plasmid in type D strain JGS1721 (accession number: ABOO01000094), and on a $\sim 12 \mathrm{~kb}$ bacteriocin-encoding plasmid in type A enterotoxigenic SM101 strains (accession number: NC_008264). The length of these putative rep genes was $\sim 1,200 \mathrm{bp}$, while that of a pIP404-rep homologous gene (PBCN30) on pBCNF5603 was smaller than other putative rep genes by $\sim 1,000 \mathrm{bp}$, with truncation at the 3' end because of an insertion (deletion) producing a stop codon at a later site. Interestingly, a $\sim 1,000$ bp sequence at the 5 ' end of these putative rep genes was very similar (percent identities of nucleotides among the rep genes of more than $90 \%$; ranging from 91.4 to $98.0 \%$ ), while $\sim 200 \mathrm{bp}$ sequences on the 3' ends of these genes were less conserved (percent identities of 57.9-84.7 \%).

\section{Compatibility between $\mathrm{pCP} 13$ and constructed recombinant plasmids carrying the identified replication region}

Each set of twenty-five chloramphenicol-resistant colonies from second-passaged cultures of strain 13, which were introduced by recombinant plasmids, pKZ210 (carrying PCP63 homolog and its upstream region) or pKZ200 (carrying the parA and parB genes, and putative parS region), were investigated (Fig. 2a). No chloramphenicol-resistant colonies showed a positive reaction in the PCR assay for the $c p b 2$ gene, and these investigated colonies also showed a negative reaction in the PCR assay for the topA gene or the cna gene on pCP13; this was likely to have occurred to cure the $c p b 2$-encoding plasmid, $\mathrm{pCP} 13$, in strain 13derived colonies introduced by one of the recombinant plasmids that carry the identified replication region with or without a putative partitioning region.

\section{Stability of the recombinant plasmid carrying the identified replication region and a putative partitioning region}

The putative partitioning region of pBCNF5603 contains two genes, parA (a putative ATPase gene) and parB (centromere-binding protein gene), and a putative parS sequence (Fig. 2a). The parA and parB genes were found on five other plasmids that harbor the identified rep gene homolog, and those gene products had more than $96 \%$ identity in terms of substituted amino acids
(Table 2). A putative parS sequence contained IR1, IR2, and one repeated sequence (Additional file 3: Figure S2), and the sequences of the parS region were almost identical among six plasmids; that is, these plasmids are likely to carry a similar plasmid replication and partitioning region to that of pBCNF5603. These findings also indicate that a putative partitioning system on these plasmids might be the parABS system.

To investigate the contribution of the putative partitioning system on pBCNF5603 to plasmid stability, the fraction carrying the recombinant plasmid, pKZ200, was monitored without any selection in two different $C$. perfringens hosts. In transformants derived from C. perfringens strains, ATCC3624 and strain 13, the proportion of chloramphenicol-resistant colonies dropped to $40 \%$ after 60 generations without antibiotic selection, while it was more than $80 \%$ under antibiotic pressure (Table 3). Therefore, the recombinant plasmid was considered to be unstable in both strains; all transformants from both strains showed high plasmid loss in terms of the proportion of plasmid-free cells. Moreover, there was no clear relationship between the host strain type and the stability of the recombinant plasmid, while strain 13 originally harbored an indigenous pCP13 that carries the closely related replication and partioning region of pBCNF5603.

In contrast to the stability of the recombinant plasmid in C. perfringens strains, an indigenous plasmid in the original strain, pBCNF5603 in F5603 or pCP13 in strain 13 , was extremely stable; after 60 generations, no indigenous plasmid-cured colonies were detected by PCR assays for two genes: the rep and the $b c n 5603$ or $c p b 2$ genes, respectively (Table 3 ).

\section{Discussion}

Bacteriocins could contribute to diseases caused by C. perfringens, for example, promoting intestinal colonization [5]. Of several identified C. perfringens bacteriocins, the most investigated one is $\mathrm{BCN} 5$ in the type A cpe-negative strain CPN50. BCN5 production is induced by stress, such as UV irradiation, and its gene is encoded by the wellstudied plasmid pIP404, which has been completely sequenced and for which the gene for plasmid replication was identified [15]. From the results in the current study, a plasmid named pBCNF5603, which does not encode toxins, was found to co-exist with the cpe/cpb2 toxin gene encoding plasmid pCPF5603 in the enterotoxigenic type A strain F5603. pBCNF5603 carries the putative bacteriocin gene $b c n 5603$ and its regulatory sequences, as determined from the results of complete sequence analysis (Fig. 1) (Additional file 1: Table S2). Of all C. perfringens bacteriocin genes, only the genetic background of the bacteriocin (BCN5) gene $(b c n)$ found on pIP404 in the type A CPN50 strain has been carefully investigated $[16,17]$. The same as the bcn gene on pIP404, the bcn5603 gene had 
Table 3 Stability of a recombinant plasmid, pKZ200, and plasmids, pBCNF5603 and pCP13, in C. perfringens strains

Incidence of colonies carrying a recombinant plasmid, pKZ200, in ATCC3624 strain

\begin{tabular}{llc}
\hline & & Rate of Cp resistant colonies \\
\hline clone 1 & Culture with Cp & $92 \%$ \\
& Culture without Cp & $40 \%$ \\
clone 2 & Culture with Cp & $84 \%$ \\
& Culture without Cp & $44 \%$ \\
\hline
\end{tabular}

Incidence of colonies carrying a ecombinant plasmid, pKZ200, in strain 13

\begin{tabular}{llc}
\hline & & Rate of Cp resistant colonies \\
\hline clone 1 & Culture with Cp & $80 \%$ \\
& Culture without Cp & $40 \%$ \\
clone 2 & Culture with Cp & $96 \%$ \\
& Culture without Cp & $44 \%$ \\
\hline
\end{tabular}

Incidence of colonies carrying a bacteriosin gene (bcn5603)-encoding plasmid, pBCNF5603, in F5603 strain

\begin{tabular}{clc} 
& & Rate of PCR positive colonies ${ }^{*}, ~ \#$ \\
\hline clone 1 & PCR for the rep gene & $100 \%$ \\
& PCR for the bcn5603 gene & $100 \%$ \\
clone 2 & PCR for the rep gene & $100 \%$ \\
& PCR for the bcn5603 gene & $100 \%$
\end{tabular}

Incidence of colonies carrying the beta2 toxin gene-encoding plasmid, pCP13, in strain 13

\begin{tabular}{clc}
\hline & & Rate of PCR positive colonies ${ }^{*}$, \# \\
\hline clone 1 & PCR for the PCP63 gene & $100 \%$ \\
& PCR for the cP62 gene & $100 \%$ \\
clone 2 & PCR for the PCP63 gene & $100 \%$ \\
& PCR for the cP62 gene & $100 \%$
\end{tabular}

Culture samples with or without $10 \mu \mathrm{g} / \mathrm{ml}$ chloramphenicol were collected after $\sim 60$ generations

*: Rate of randomly selected colonies

\#: To investigate the presence of pBCNF5603 in F5603 strain or pCP13 in strain 13, randomly selected colonies were tested with colony PCR assay for three genes, rep (PCP63), bcn5603, and cpb2 genes. Colony PCR reactions were performed under the same conditions as described in the plasmid compatibility section

regulatory $u v i A$ and $u v i B$ genes and regulatory sequences, such as promoter sequences and the LexA-binding sequence. Moreover, these regulatory sequences were almost identical to the respective sequences on pIP404. In fact, $C$. perfringens F5603 strain was likely to produce a bacteriocin, which was promoted by UV irradiation, using pCP13-cured strain 13 as an indicator strain. Moreover, transcription of the $b c n 5603$ gene was likely to be promoted by the addition of mitomycin $\mathrm{C}$ (data not shown); that is, the extreme conservation of the $b c n$ gene cluster on pBCNF5606 indicates that production of the BCN5603 bacteriocin might be induced in the same manner as for BCN5 in the CPN50 strain.
Comparative genomics using the GenBank database indicated that $b c n 5603$ gene homologs have been found in several other virulent strains. These homologs encode putative bacteriocins that carry commonly conserved domains, namely, SH3 and peptidase M13 family domains. Moreover, a recent PCR survey indicated that BCN5 gene homologs are found in cpe-positive type A human disease isolates (F4013 and NCTC8798), as well as type $\mathrm{B}, \mathrm{D}$, and $\mathrm{E}$ animal disease isolates [22]. Taking these findings together, the production of this group of bacteriocins by $C$. perfringens virulent strains might benefit for them compared with other enteric bacteria in the human or animal gut, so this group of bacteriocins might contribute to the virulence of $C$. perfringens strains.

To date, only two kinds of rep gene have been identified on $C$. perfringens plasmids: the rep gene on the antibiotic resistance $\mathrm{pCW} 3$ plasmid and the rep gene on the bacteriocin gene-encoding pIP404 [15, 23]. The rep gene of the most representative toxin gene-encoding plasmids is highly similar to the rep gene of pCW3 $[8,23]$. To understand the relationship between pBCNF5603 and other plasmids, especially plasmids carrying the previously identified rep genes, the replication region of pBCNF5603 was identified. On the basis of sequence in-

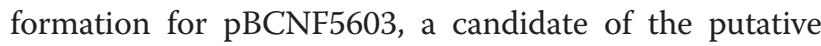
replication region, which is similar to the putative pCP13 plasmid replication region, was identified, while the rep gene of pCP13 has not yet been identified. The results of investigating the replication ability of the PBCN16 region indicated that recombinant plasmids (pKZ200 and pKZ210), both of which carry PBCN16 and its upstream region, were replicable in two transformable $C$. perfringens strains, ATCC3624 and strain 13; the similarly organized region of pBCNF5603 with that of $\mathrm{pCP} 13$ is most likely to be the replication region that must contain the rep gene. Moreover, five inverted repeated sequences, probably iterons, and an AT-rich region were also identified upstream of the PBCN16 ORF; PBCN16 is likely to be the rep gene of pBCNF5603, and therefore pBCNF5603 might be an iteron-containing plasmid (ICP). By comparative genomics using the GenBank database, PBCN16 homologs were found in several other plasmids including pCP13, pCP-OS1, and pCP-TS1, which are toxin-encoding plasmids other than the prototypes of toxin-encoding plasmid [20]. Moreover, these plasmids carry upstream iteron-like sequences that are highly similar to those sequences of PBCN16 (Fig. 3). Therefore, the plasmid family that carries the PBCN16 homolog in the putative replication region is also likely to be ICP.

Prototypes of toxin-encoding plasmids carry the common replication and partitioning system, the parMRC system, and these systems might be related to plasmid compatibility [23, 24]. In the same C. perfringens cells of the F5603 strain, the bacteriocin-encoding plasmid 
pBCNF5603, which carries a different replication and partitioning system, the parABS system, can co-exist with one of the prototypes of toxin-encoding plasmids, cpe-encoding plasmid pCPF5603. On the other hand, recombinant plasmids carrying the identified rep gene and upstream iteron sequences of pBCNF5603 were likely to induce pCP13 curing in strain 13; pBCNF5603 and pCP13 might be incompatible plasmids, possibly because of iteron-based incompatibility. In general, two components, Rep and iterons, lie at the center of all models of ICP replication control, so replication-mediated incompatibility has been used to classify plasmids [7]. Taking these findings together, pBCNF5603 and pCP13 are likely to be classified into the same Inc group, but different groups from the prototypes of toxin-encoding plasmid. Moreover, on the basis of the high similarity of upstream iteron sequences of the putative rep gene, recently sequenced plasmids, pCP-TS1, pCP-OS1, and other plasmids (pF262B and plasmid in WAL-14572 strain), are likely to belong to the pBCNF5603 Inc family [20,21].

A recombinant plasmid named pKZ100, which carries pIP404 rep homolog, PBCN30, is non-functional in $C$. perfringens strain, while $\mathrm{PBCN} 30$ was also identified as a candidate of the rep gene on pBCNF5603 by a BLAST search (Fig. 2b). On the other hand, pIP404 rep gene homolog on a small plasmid, pCP8533S12, in type B strain NCTC8533 is functional based on the transformation experiment results obtained using another recombinant plasmid, pKZ110. By comparative genomics using the GenBank database, pIP404 rep gene homologs were also found in several strains that also harbor prototypes of toxin-encoding plasmid(s). Comparing the sequence of these rep homologs in different strains, PBCN30 on pBCNF5603 was $\sim 200$ bp smaller than other homologs; in addition, PBCN30 on pBCNF5603 has a point mutation and, as a result, may have become non-functional (Fig. 2b). From these findings, it was suggested that $\sim 200 \mathrm{bp}$ on the 3 ' side of this type of rep gene might be important for plasmid replication.

Upstream of the PBCN16 rep gene, the putative plasmid partitioning-related genes (parA, parB, and maybe PCP03 homolog) and a putative parS sequence were identified (Fig. 2a). A similarly organized putative partitioning region was also found on pCP13, pCP-OS1, pCP-TS1, pF262, and plasmid in the WAL-14572 strain. Therefore, the highly homologous rep gene (more than $90 \%$ identity) and a putative partitioning region are likely to be a core region of these plasmids. In general, the partitioning region has a central role in plasmid segregation and is thus important for plasmid stability [25]. To investigate the contribution of the putative partitioning region of pBCNF5603 to plasmid stability, the stability of the recombinant plasmid that carries the replication and partitioning region was evaluated in two electro-transformable strains, ATCC3624 and strain 13. Strain 13 originally carries the indigenous plasmid, $\mathrm{pCP} 13$, which has a replication and partitioning region that is closely related to that of pBCNF5603, while the ATCC3624 strain naturally harbors no plasmid. Although strain 13 and F5603 strain stably carried pCP13 or pBCNF5603, respectively, the recombinant plasmid that carries the pBCNF5603-derived replication and partitioning region was relatively unstable, even in pCP13-free strain 13-derived strain, as in the ATCC3624 strain. These findings might indicate that the identified replication and partitioning region of pBCNF5603 is insufficient for plasmid stability, while the replication and partitioning region is important for plasmid stability [25]. The possible reasons for recombinant plasmid instability are as follows: 1) the influence of the integrity of iteron sequences and arrangement; 2) the sequence of the genes in this region might be strictly controlled: the integrity of these regions might be important for plasmid stability; and 3) the gene(s) encoding hypothetical or conserved hypothetical protein on pBCNF5603 could have an important role in the stability of the plasmid. In addition to these possibilities, host factors could possibly promote plasmid stability; the replication and partitioning region might co-operate with host chromosomal factors [25]. Strain 13 is likely to have a chromosomal genetic background that is more similar to the F5603 strain than to the ATCC3624 strain, given previous MLST results [22]. However, the stability levels of the recombinant plasmid that carries the replication and partitioning region were very similar in those two $C$. perfringens strains. Therefore, the contribution of the host factors to the stability of the recombinant plasmid could differ compared with plasmids in E. coli.

Interestingly, a similarly organized putative plasmid partitioning region, the parABS region, was found on a $\beta$ toxin gene $(c p b)$-encoding plasmid, pCP8533cbp, in the type $\mathrm{B}$ NCTC8533 strain (accession number: AB736083) (Fig. 2a). By comparative genomics using the GenBank database, these conserved parABS regions were also identified on the $c p b$ plasmids in the type B ATCC3626 strain (accession number: NZ_ABDV01000024) and in the type C JGS1945 strain (accession number: NZ_ABDU01000064). However, these $c p b$-encoding plasmids did not harbor a PCP63 homolog on the upstream region of the parA gene. The parABS region on these $c p b$-encoding plasmids could be related to plasmid properties, such as partitioning elementbased plasmid incompatibility.

To our knowledge, this is the first report about identification of the rep gene of a bacteriocin-encoding plasmid in enterotoxigenic $C$. perfringens strain. The rep gene identified in the current study is unique and might be encoded by important plasmids because the identified rep gene homologs were found on not only the bacteriocinencoding plasmid, but also toxin-encoding plasmids. In 
addition, $C$. perfringens plasmids that carry the identified rep gene or its homolog might be the same Inc family plasmids.

\section{Conclusions}

The current study demonstrated that i) the replication region of bacteriocin gene encoding plasmid, pBCNF5603 in enterotoxigenic $C$. perfringens strain was identified, ii) the plasmid carrying the identified rep gene are predicted as a iteron-containing plasmid (ICP), iii) the identified replication region are also found on toxin gene encoding plasmid in virulent strains, e.g. BEC (binary enterotoxin of C. perfringens) gene encoding plasmids, iv) the identified replication region contributes plasmid compatibility; plasmids carrying the homologs with the identified rep gene should belong to the same Inc family, v) the plasmid replication and the predicted plasmid partitioning regions are insufficient for stable maintenance of recombinant plasmid which carry those of pBCNF5603 in C. perfringens strains.

Collectively, findings in the current study provide the important insights of the bacteriocin gene and toxin gene encoding plasmids in virulent $C$. perfringens strains; contribution of the replication region to plasmid compatibility have not been investigated in previous studies, while the replication region of a few plasmids in virulent $C$. perfringens strains was reported. Moreover, role of the plasmid replication and partitioning regions on C. perfringens plasmid stability have not been investigated, although the important plasmids are likely to be highly stable in virulent strains. Further detailed studies on the issues investigated in the current study should help in understanding plasmid biology in virulent $C$. perfringens strains.

\section{Additional files}

Below is the link to the electronic supplementary material.

Additional file 1: Table S1. PCR primers for amplification of putative replication regions and used for the detection of genes with colony PCR assays. Table S2. Homology search results of putative ORFs on pBCNF5603. Table S3. Homology search results of putative ORFs on pCP8533S12.

Additional file 2: Figure S1. PFGE analysis of plasmids from C. perfringens F5603 strain. Agarose plug containing genomic DNA from F5603 strains was subjected to PFGE and stained with ethidium bromide (Wako Pure Chemical, Osaka, Japan). M: Low Range PFG marker (NEB, Tokyo, Japan).

Additional file 3: Figure S2. Repeated elements on the putative parS region. The arrow-headed bars depict the two pairs of inverted repeats (IR1 and IR2). And green colored regions depict two short-repeated sequences.

\section{Competing interests}

The authors declare that they have no competing interests.

\section{Authors' contribution}

KM carried out most of the experiments, and participated in the discussions on the study design, analysis and interpretation of the data, and in the writing of the manuscript. MN, MO, MT participated in the discussions on the study design, analysis and interpretation of the data. $\mathrm{KM}$, KO participated in the sequence alignment analysis and interpretation of sequence data of pBCNF5603 and pCP13 (partial sequence). KM, SS TT carried out the plasmid compatibility and plasmid stability experiments. All authors read and approved the final manuscript.

\section{Acknowledgements}

This work was supported by a Grant-in-Aid for Scientific Research from the Ministry of Education, Culture, Sports, Science, and Technology of Japan, 2014.

Sequence data from this article have been deposited in the GenBank database with the accession numbers AB189671, AB736082, AB736083, and LC011526.

\section{Author details}

'Department of Microbiology, Faculty of Pharmaceutical Sciences, Tokushima Bunri University, Yamashiro-cho 180, Tokushima 770-8514, Japan. ${ }^{2}$ Division of Microbiology and Infectious Diseases, Niigata University Graduate School of Medical and Dental Sciences, 2-5274, Gakkocho-dori, Chuo-ku, Niigata 951-8514, Japan.

Received: 13 December 2014 Accepted: 12 May 2015

Published online: 09 June 2015

\section{References}

1. McClane BA, Robertson SL, Li J. Clostridium perfringens. In: Doyle MP, Buchanan RL, editors. Food Microbiology :Fundamentals and Frontiers. 4th ed. Washington, D.C: ASM press; 2013. p. 465-90.

2. Amimoto $K$, Noro T, Oishi E, Shimizu M. A novel toxin homologous to large clostridial cytotoxins found in culture supernatant of Clostridium perfringens type C. Microbiol. 2007;153:1198-206.

3. Keyburn AL, Boyce JD, Vaz P, Bannam TL, Ford ME, Parker D, et al. NetB, a new toxin that is associated with avian necrotic enteritis caused by Clostridium perfringens. PLoS Pathog. 2008:4:e26.

4. Petit L, Gibert M, Popoff MR. Clostridium perfringens: toxinotype and genotype Trend Microbiol. 1999;7:104-10.

5. Barbara AJ, Trinh HT, Glock RD, Songer GJ. Necrotic enteritis-producing strains of Clostridium perfringens displace non-necrotic enteritis strains from the gut of chicks. Vet Microbiol. 2008;126:377-82.

6. Tagg JR, Dajani AS, Wannamaker LW. Bacteriocins of gram-positive bacteria. Microbiol Mol Biol Rev. 1976:40:722-56.

7. Kruger R, Pakowski SA, Filutowicz M. Participating elements in the replication of Iteron-containing plasmids. In: Funnell BE, Phillips GJ, editors. Plasmid Biology. Washington D. C: ASM Press; 2004. p. 25-45.

8. Miyamoto K, Fisher DJ, Li J, Sayeed S, Akimoto S, McClane BA. Complete sequencing and diversity analysis of the enterotoxin-encoding plasmids in Clostridium perfringens type A non-food-borne human gastrointestinal disease isolates. J Bacteriol. 2006:188:1585-98.

9. Miyamoto K, Li J, Sayeed S, Akimoto S, McClane BA. Sequencing and diversity analyses reveal extensive similarities between some epsilon-toxin-encoding plasmids and the pCPF5603 Clostridium perfringens enterotoxin plasmid. J Bacteriol. 2008;190:7178-88.

10. Shimizu T, Ohtani K, Hirakawa H, Ohshima K, Yamashita A, Shiba T, et al. Complete genome sequence of Clostridium perfringens, an anaerobic flesh-eater. Proc Natl Acad Sci USA. 2002;99:996-1001.

11. Miyamoto K, Yumine N, Mimura K, Nagahama M, Li J, McClane BA, et al. Identification of novel Clostridium perfringens type $\mathrm{E}$ strains that carry an iota toxin plasmid with a functional enterotoxin gene. PLoS One. 2011;6:e20376.

12. Sloan J, Warner TA, Scott PT, Bannam TL, Berryman DI, Rood Jl. Construction of a sequenced Clostridium perfringens-Escherichia coli shuttle plasmid. Plasmid. 1992;27:207-19.

13. Sarker MR, Carman RJ, McClane BA. Inactivation of the gene (cpe) encoding Clostridium perfringens enterotoxin eliminates the ability of two cpe-positive C. perfringens type A human gastrointestinal disease isolates to affect rabbit ileal loops. Mol Microbiol. 1999;33:946-58.

14. Gelder LD, Ponciano JM, Joyce P, Top EM. Stability of a promiscuous plasmid in different hosts: no guarantee for a long-term relationship. Microbiol. 2007;153:452-63.

15. Rood J, Cole ST. Molecular genetics and pathogenesis of Clostridium perfringens. Microbiol Mol Biol Rev. 1991;55:621-48. 
16. Dupuy B, Raffestin S, Matamouros S, Mani N, Popoff MR, Sonenshein AL. Regulation of toxin and bacteriocin gene expression in Clostridium by interchangeable RNA polymeraser sigma factors. Mol Microbiol. 2006;60:1044-57.

17. Dupuy B, Mani N, Katayama S, Sonenshein AL. Transcription activation of a UV-inducible Clostridium perfringens bacteriocin gene by a novel $\sigma$ factor. Mol Microbiol. 2005;55:1196-206.

18. Paulsen IT, Banerjei L, Myers GSA, Nelson KE, Seshadri R, Read TD, et al. Role of mobile DNA in the evolution of vancomycin-resistant Enterococcus faecalis. Science. 2003;299:2071-4.

19. Tettelin H, Masignani V, Cieslewicz MJ, Eisen JA, Peterson S, Wessels MR, et al. Complete genome sequence and comparative genomic analysis of an emerging human pathogen, serotype V Streptococcus agalactiae. Proc Natl Acad Sci USA. 2002;99:12391-6.

20. Yonogi S, Matsuda S, Kawai T, Yoda T, Harada T, Kumeda Y, et al. BEC, a novel enterotoxin of Clostridium perfringens found in human clinical isolates from acute gastroenteritis outbreaks. Infect Immun. 2014;82:2390-9.

21. Nowell VJ, Kropinski AM, Songer JG, Maclnnes JI, Parreira VR, Prescott JF. Genome sequencing and analysis of a type A Clostridium perfringens isolate from a case of bovine clostridial abomasitis. PloS One. 2012;7:e32271.

22. Deguchi A, Miyamoto K, Kuwahara T, Miki Y, Kaneko I, Li J, et al. Genetic characterization of type A enterotoxigenic Clostridium perfringens strains. PLoS One. 2009:4:e5598.

23. Bannam TL, Teng WL, Bulach D, Lyras D, Rood Jl. Functional identification of conjugation and replication regions of the tetracycline resistant plasmid pCW3 from Clostridium perfringens. J Bacteriol. 2006;188:4942-51.

24. Parreira VR, Costa M, Eikmeyer F, Blom J, Prescott JF. Sequence of two plasmids from Clostridium perfringens chicken necrotic enteritis isolates and comparison with C. perfringens conjugative plasmids. PLos One. 2012;7:e49753.

25. Funnell BE, Slavcev DA. Partitioning systems of bacterial plasmids. In: Funnell BE, Phillips GJ, editors. Plasmid Biology. Washington D.C: ASM Press; 2004. p. 81-103.

\section{Submit your next manuscript to BioMed Central and take full advantage of:}

- Convenient online submission

- Thorough peer review

- No space constraints or color figure charges

- Immediate publication on acceptance

- Inclusion in PubMed, CAS, Scopus and Google Scholar

- Research which is freely available for redistribution 